\title{
Foxc2 induces Wnt4 and Bmp4 expression during muscle regeneration and osteogenesis
}

\author{
MC Gozo ${ }^{1,5}$, P-J Aspuria ${ }^{1}$, D-J Cheon ${ }^{1}$, AE Walts ${ }^{2}$, D Berel ${ }^{3}$, N Miura ${ }^{4}$, BY Karlan ${ }^{1}$ and S Orsulic ${ }^{\star 1}$
}

Proliferation and fusion of myoblasts is a well-orchestrated process occurring during muscle development and regeneration. Although myoblasts are known to originate from muscle satellite cells, the molecular mechanisms that coordinate their commitment toward differentiation are poorly understood. Here, we present a novel role for the transcription factor Forkhead box protein C2 (Foxc2) in regulating proliferation and preventing premature differentiation of activated muscle satellite cells. We demonstrate that Foxc2 expression is upregulated early in activated mouse muscle satellite cells and then diminishes during myogenesis. In undifferentiated $\mathrm{C} 2 \mathrm{C} 12$ myoblasts, downregulation of endogenous Foxc2 expression leads to a decrease in proliferation, whereas forced expression of FOXC2 sustains proliferation and prevents differentiation into myotubes. We also show that FOXC2 induces Wnt signaling by direct interaction with the Wnt4 (wingless-type MMTV integration site family member-4) promoter region. The resulting elevated expression of bone morphogenetic protein-4 (Bmp4) and RhoA-GTP proteins inhibits the proper myoblast alignment and fusion required for myotube formation. Interestingly, continuous forced expression of FOXC2 alters the commitment of $\mathrm{C} 2 \mathrm{C} 12$ myoblasts toward osteogenic differentiation, which is consistent with FOXC2 expression observed in patients with myositis ossificans, an abnormal bone growth within muscle tissue. In summary, our results suggest that (a) Foxc2 regulates the proliferation of multipotent muscle satellite cells; (b) downregulation of Foxc2 is critical for myogenesis to progress; and (c) sustained Foxc2 expression in myoblast cells suppresses myogenesis and alters their lineage commitment toward osteogenesis by inducing the Wnt4 and Bmp4 signaling pathways.

Cell Death and Differentiation (2013) 20, 1031-1042; doi:10.1038/cdd.2013.34; published online 3 May 2013

Muscle satellite cells are resident stem cells localized between the basal lamina and the sarcolemma of myofibers. During postnatal development, these satellite cells provide myonuclei for growth of skeletal muscle. In adult skeletal muscles, satellite cells comprise $1-4 \%$ of myofibers and remain quiescent ${ }^{1,2}$ unless involved in homeostasis, hypertrophy or muscle repair at which time they become activated, proliferate and produce the myoblasts needed to replace or repair myofibers. ${ }^{2,3}$ Deregulation of factors involved in the maintenance of muscle homeostasis and in the differentiation of muscle precursors can lead to a variety of pathologic conditions, including muscular dystrophies and myositis ossificans or heterotopic ossification. ${ }^{4,5}$ The role of muscle satellite cells in the pathology of these disorders is not well understood. Identification of key regulators and elucidation of the processes involved in satellite cell activation and differentiation could provide important insights into the etiology, treatment and/or prevention of muscle disorders.

Recent studies have shown that bone morphogenetic proteins (BMPs) are important in the activation and proliferation of muscle satellite cells, and in the prevention of premature myogenic differentiation. ${ }^{6,7}$ BMPs are members of the transforming growth factor- $\beta$ (TGF- $\beta$ ) family that initiate signaling by binding to transmembrane type- 1 and type- 2 BMP receptors (BMPRs). Upon ligand binding, transphosphorylation of type-1 by type-2 receptors activates the receptor-regulated Smad (Sma and Mad-related protein) transcription factors (R-Smads) - Smad1, Smad5 and Smad8 (pSmad1/5/8) - to assemble with Smad4 and translocate to the nucleus. These Smad complexes control the transcription of several regulatory elements, including some of the inhibitor of differentiation (Id) proteins. Binding of these Id proteins to ubiquitously expressed E-proteins prevents the activation of the muscle-specific transcription factors myogenic differentiation-1 (MyoD) and myogenin, and impedes myogenic differentiation. ${ }^{8}$ Previous studies have shown that intracellular BMP signaling through BMPR-1A is highly elevated in proliferating satellite cells and the effect of stimulating BMP signaling with the addition of BMP4 results in an increased number of activated satellite cells and a decreased number of

\footnotetext{
${ }^{1}$ Women's Cancer Program at the Samuel Oschin Comprehensive Cancer Institute, Los Angeles, CA, USA; ${ }^{2}$ Department of Pathology and Laboratory Medicine, Cedars-Sinai Medical Center, Los Angeles, CA, USA; ${ }^{3}$ Department of Biostatistics at the Samuel Oschin Comprehensive Cancer Institute, Cedars-Sinai Medical Center, Los Angeles, CA, USA; ${ }^{4}$ Department of Biochemistry, Hamamatsu University School of Medicine, Hamamatsu, Japan and ${ }^{5}$ Graduate Program in Biomedical Science and Translational Medicine, Cedars-Sinai Medical Center, Los Angeles, CA, USA

*Corresponding author: S Orsulic, Women's Cancer Program at the Samuel Oschin Comprehensive Cancer Institute, Cedars-Sinai Medical Center, 8700 Beverly Blvd., Suite 290W, Los Angeles, CA 90048, USA. Tel: 310423 9546; Fax: 310423 9753; E-mail: Sandra.Orsulic@cshs.org

Keywords: Foxc2; Bmp4; Wnt4; myogenesis; osteogenesis

Abbreviations: ALP, alkaline phosphatase; BMP2, bone morphogenetic protein-2; Bmp4, bone morphogenetic protein-4; BMPR-1A, bone morphogenetic receptor type-1A; CTX, Najamossambica cardiotoxin; DVL, dishevelled, dsh homolog-1; Foxc2, Forkhead box C2; MyoD, myogenic differentiation-1; Pax7, paired box homeotic-7; PCP, Wnt-planar cell polarity signaling pathway; RhoA, ras homolog family member-A; Runx2, Runt-related transcription factor-2; Smad1 and -5, Sma and Mad-related protein- 1 and 5 ; TGF- $\beta$, transforming growth factor- $\beta$; Wnt 4 , wingless-type MMTV integration site family member-4

Received 13.9.12; revised 18.3.13; accepted 26.3.13; Edited by RA Knight; published online 03.5.13
} 
differentiating myoblasts. ${ }^{7}$ Although Bmp4 has been specifically shown to be important in regulating myogenic and osteogenic progenitor cells that reside in skeletal muscle, ${ }^{6,9,10}$ the factors that regulate Bmp signalling during muscle regeneration have yet to be identified.

The Wnt signaling pathway has also been implicated in maintaining the satellite stem cell pool. ${ }^{11}$ Wnt signaling is inhibited by myostatin, another member of the TGF- $\beta$ superfamily and a major inhibitor of skeletal muscle growth. Elevated levels of the Wnt4 (wingless-type MMTV integration site family member-4) ligand have been observed in mice lacking myostatin and in vitro studies have shown that Wnt4 overexpression stimulates muscle satellite cell proliferation in vitro. ${ }^{12}$ Since binding of the Wnt ligand to its receptor can activate signaling via $\beta$-catenin $(\beta \mathrm{C})$-dependent or $\beta \mathrm{C}$ independent pathways, it can affect the regulation of diverse cellular processes, including muscle satellite cell determination, proliferation and differentiation. ${ }^{13}$ Cross-talk between the TGF- $\beta / \mathrm{Bmp}$ and the Wnt signaling pathways can reciprocally regulate their ligand production as part of several developmental processes. ${ }^{14}$ It has also been demonstrated that the nuclear Smad- $\beta \mathrm{C}$-Lef protein complex regulates a host of shared target genes in a synergistic manner. ${ }^{15,16}$ Therefore, identification of the upstream regulators of the Wnt and Bmp pathways is important in understanding the intracellular signals that regulate the proliferation and differentiation of myoblasts.

Forkhead box (Fox) proteins comprise a family of transcription factors that are involved in the regulation of cell growth, proliferation, differentiation and longevity. ${ }^{17}$ Previous studies have shown that Fox protein-C2 (Foxc2), a member of the ' $\mathrm{C}$ ' subfamily, plays a role in mesenchymal tissue differentiation. Foxc2 inhibits differentiation of white adipocytes and is required for proper bone formation during embryonic development and osteoblastogenesis in adult bone precursor cells. ${ }^{18,19}$ In this study, we investigate the role of Foxc2 in the proliferation and differentiation of muscle satellite cells, specifically the role of Foxc2 during muscle regeneration in response to injury. We present a novel mechanism where Foxc2 plays a role in maintaining the proliferative state of undifferentiated myoblasts by regulating Wnt4 signaling, which, in turn, induces Bmp4 and RhoA-GTP protein expression. We demonstrate that, similar to its downstream target Bmp4, overexpression of Foxc2 inhibits myogenesis and directs the commitment of $\mathrm{C} 2 \mathrm{C} 12$ myoblasts and NIH3T3 fibroblasts toward osteogenesis. The expression of FOXC2 observed in areas of ectopic bone formation in samples from patients with myositis ossificans underscores the relevance of FOXC2 to human disease.

\section{Results}

Foxc2 is expressed in $\mathrm{Pax}^{+} / \mathrm{MyoD}^{+}$activated satellite cells during muscle regeneration and development. To investigate if Foxc2 is upregulated during muscle regeneration, we induced muscle damage by intramuscular injection of cardiotoxin (CTX) into the anterior tibialis and rectus femoris of 10-week-old mice. We analyzed the regenerating muscle for expression of BrdU, MyoD and Foxc2 at 4, 6 and 14 days post CTX injection (Figure 1a). We detected high
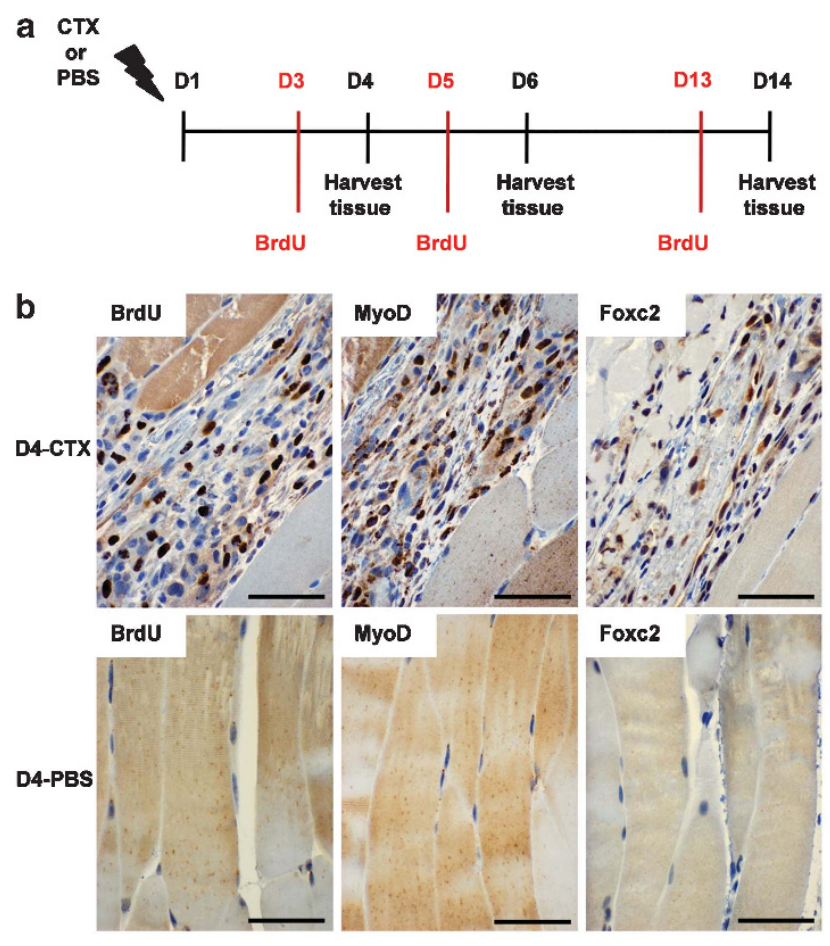

C
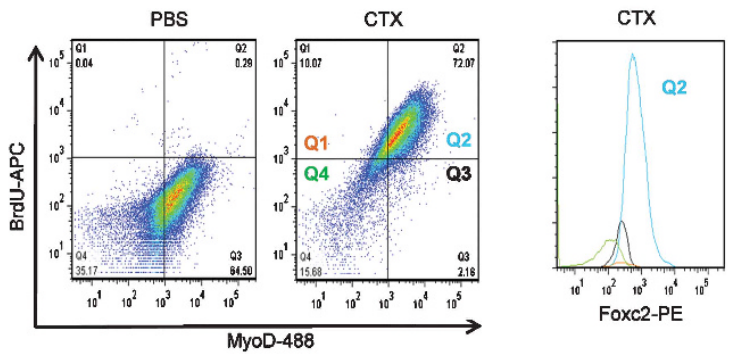

d
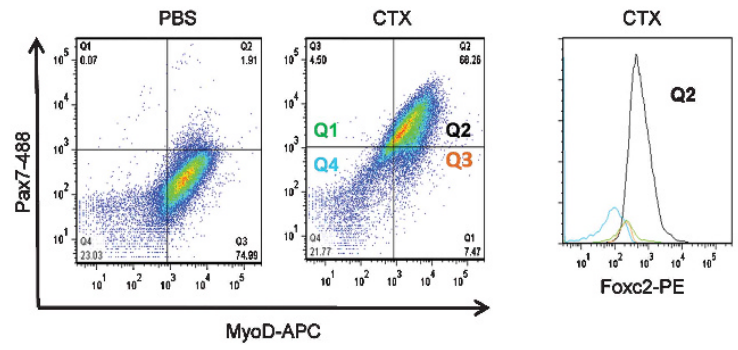

Figure 1 Foxc2 is expressed in activated muscle satellite cells during adult muscle regeneration. (a) Schematic diagram of PBS or CTX injection into the leg muscle of 10-week-old mice. Muscle was harvested 4, 6 and 14 days post injection. $\mathrm{BrdU}$ was injected intraperitoneally $24 \mathrm{~h}$ before tissue harvesting. (b) Immunohistochemistry was performed to assess the expression levels of BrdU, MyoD and Foxc2 at day 4 in the CTX- and PBS-injected mice. Images were taken with an Olympus DP25 camera with an Olympus BX43 light microscope. Scale bar, $50 \mu \mathrm{m}$. (c) A cell suspension was prepared from the harvested tissue and fixed with permeabilization/fixation buffer to measure Foxc2 expression levels in $\mathrm{BrdU}^{+}$and $\mathrm{MyoD}^{+}$cells using FACS analysis. (d) Foxc2 expression levels were measured in $\mathrm{Pax}^{+}$and $\mathrm{MyoD}^{+}$cells

levels of BrdU and MyoD at day 4 (Figure 1b) and diminished levels at days 6 and 14 (data not shown). At 4 days post CTX injection, Foxc2 expression was also detected in progenitor cells adjacent to the myofibers undergoing repair and 
regeneration (Figure 1b). Foxc2 expression was undetectable at 6 and 14 days post CTX injection. No BrdU-, MyoDor Foxc2-positive areas were detected in control PBSinjected muscle (Figure 1b). Based on these findings, we conclude that Foxc2 is only expressed early in muscle repair when satellite cell activation and proliferation occur. Fluorescence-activated cell sorting (FACS) analyses of the same tissues at day 4 showed a significant increase in $\mathrm{BrdU}^{+} /$ $\mathrm{MyoD}^{+}$muscle cell populations in the CTX-injected mice compared with the PBS-injected mice (Figure 1c). In the CTX-injected mice, Foxc2 expression levels were higher in $\mathrm{BrdU}^{+} / \mathrm{MyoD}^{+}$cells (Q2) than in $\mathrm{BrdU}^{-} / \mathrm{MyoD}^{+}$(Q3) and $\mathrm{BrdU}^{+} / \mathrm{MyoD}^{-}$cells (Q1) (Figure 1c). Analysis of the expression of the satellite cell markers paired box homeotic-7 (Pax7) and MyoD also showed increased numbers of $\mathrm{Pax}^{+}$(quiescent satellite cells) and $\mathrm{MyoD}^{+}$(myoblasts committed to differentiation) satellite cells in the CTX-injected mice compared with the PBS-injected mice (Figure 1d). We detected higher levels of Foxc2 in the $\mathrm{Pax} 7^{+} / \mathrm{MyoD}^{+}$cells (Q2) than in $\mathrm{Pax}^{+} / \mathrm{MyoD}^{-}$(Q1) or Pax7 ${ }^{-} / \mathrm{MyoD}^{+}$(Q3) cells (Figure 1d). These results demonstrate that Foxc2 is upregulated in activated muscle satellite cells and support its role in muscle regeneration. Similarly, when we assessed Foxc2 levels in Pax7- and MyoD-labeled developing muscle of 12-day-old mice, we observed the highest levels of Foxc2 in cells that co-expressed Pax7 and MyoD (Supplementary Figure S1), suggesting that Foxc2 is predominantly expressed in activated satellite cells during postnatal muscle development.

Forced expression of $\mathrm{FOXC2}$ in $\mathrm{C} 2 \mathrm{C} 12$ cells inhibits myotube formation. To determine whether Foxc2 is only expressed in undifferentiated myoblasts, we analyzed the endogenous expression levels of Foxc2 in $\mathrm{C} 2 \mathrm{C} 12$ mouse myoblasts at different time points during induced myogenic differentiation (Figure 2a). Foxc2 was highly expressed in proliferating myoblasts and its expression diminished during myogenic differentiation (Figures $2 b$ and $c$ ). To establish if Foxc2 affects the differentiation of myoblasts into myotubes, we generated stable $\mathrm{C} 2 \mathrm{C} 12$ cell lines that overexpressed human FOXC2 (designated C2C12-FOXC2 cells) or an empty vector (designated C2C12-EV cells) as a control. FOXC2 was continuously overexpressed during different stages of myogenic differentiation in $\mathrm{C} 2 \mathrm{C} 12-\mathrm{FOXC2}$ cells as shown by quantitative real-time PCR (qRT-PCR) using primers specific for human FOXC2 (Figure 2d) and western blot analysis (Figure 2e). Myogenic differentiation was evaluated by light microscopy at different time points. C2C12-FOXC2 cells failed to form myotubes (Figure 2f), indicating that Foxc2 is involved in the suppression of myogenesis.

Next, we compared C2C12-EV to C2C12-FOXC2 cells' expression levels of myogenic markers known to regulate different stages of myogenesis. As expected in C2C12-EV cells, MyoD expression levels were high in undifferentiated

a
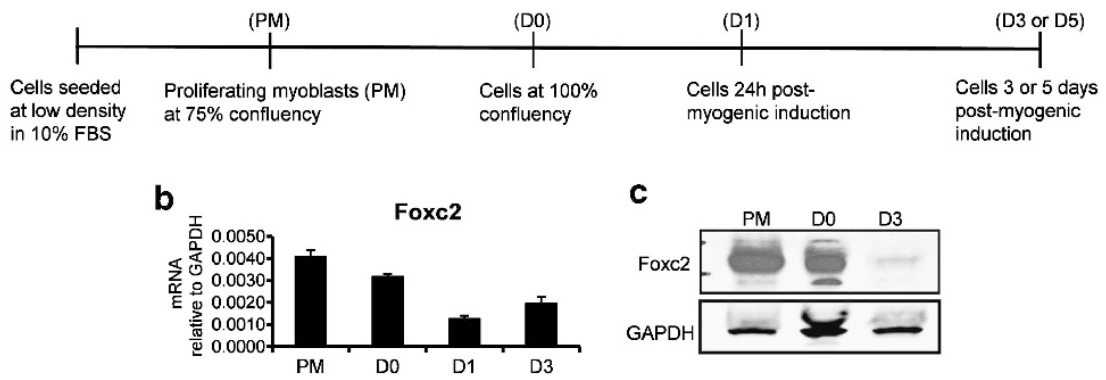

C

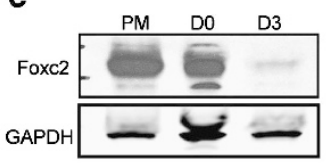

d

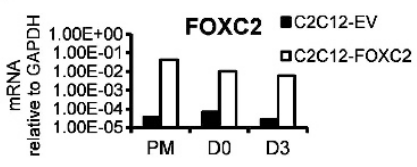

e

f

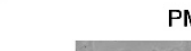

PM

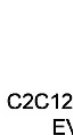

Do

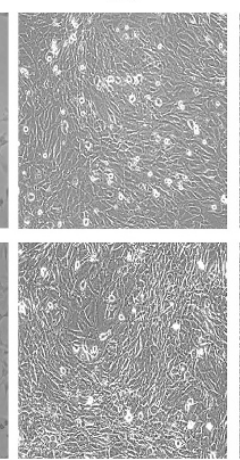

D5

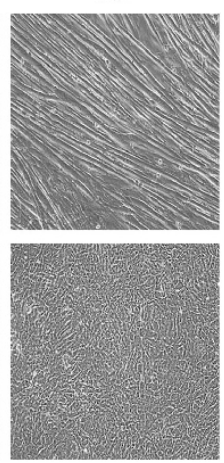

Figure 2 Endogenous Foxc2 expression diminishes during myogenic differentiation and forced expression of FOXC2 inhibits myogenic differentiation. (a) Schematic representation of the differentiation assay and the time points at which $\mathrm{C} 2 \mathrm{C} 12$ cells were analyzed. At $75 \%$ confluency, the cell culture mainly consists of proliferating myoblasts (PM). Cells were induced to undergo myogenic differentiation at 100\% confluency (D0) and maintained in differentiation media up to 5 days post confluency (D1-D5). (b) qRT-PCR was performed at different time points to assess endogenous Foxc2 mRNA levels during differentiation. (c) Western blot analysis was performed to determine Foxc2 endogenous protein levels during differentiation. (d) C2C12-EV and C2C12-FOXC2 cells were generated as stable cell lines. Cells at different stages of myogenic differentiation were analyzed by qRT-PCR using primers specific for human FOXC2. (e) Forced expression of FOXC2 during different stages of differentiation was confirmed by western blot analysis using a FOXC2 antibody. (f) Bright-field images of C2C12-EV and C2C12-FOXC2 cells were taken at different stages of differentiation. Cells were visualized at $\times 10$ magnification using an Olympus IX51 microscope 
a
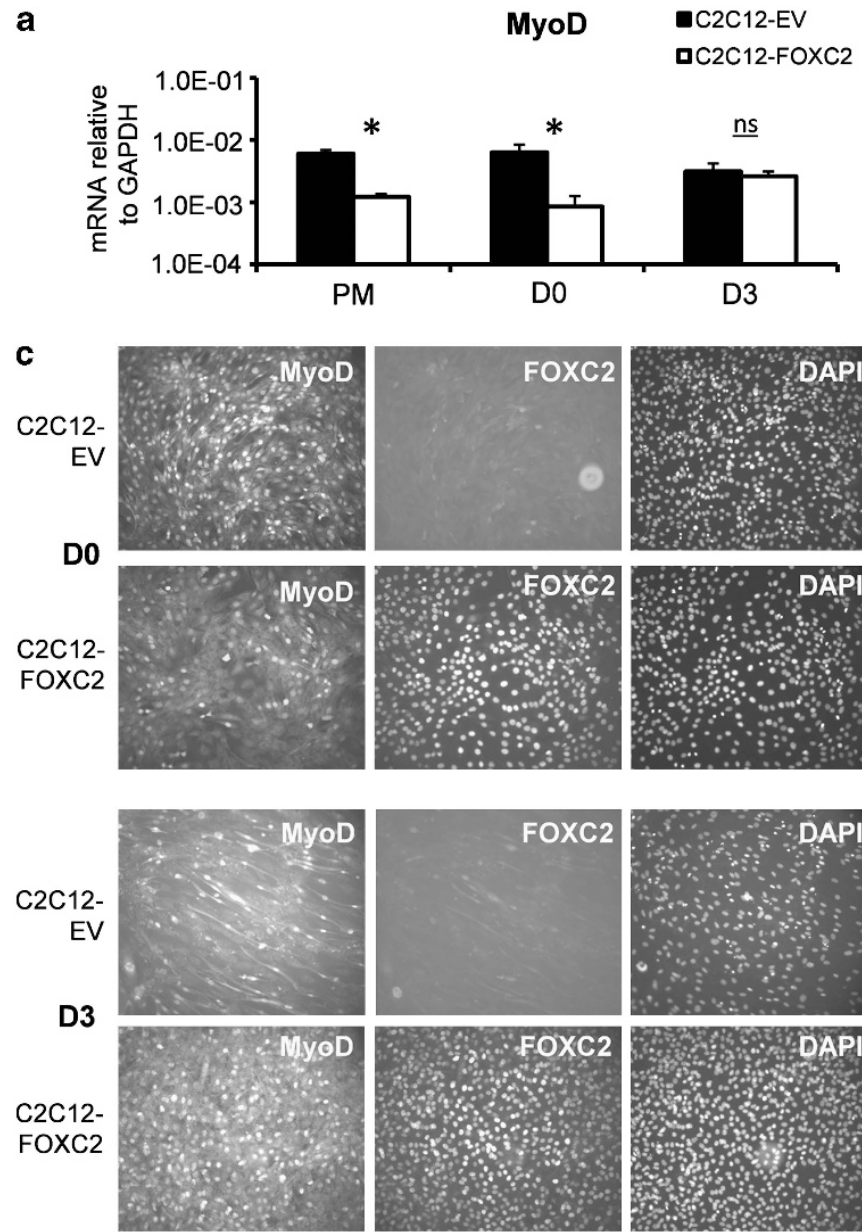
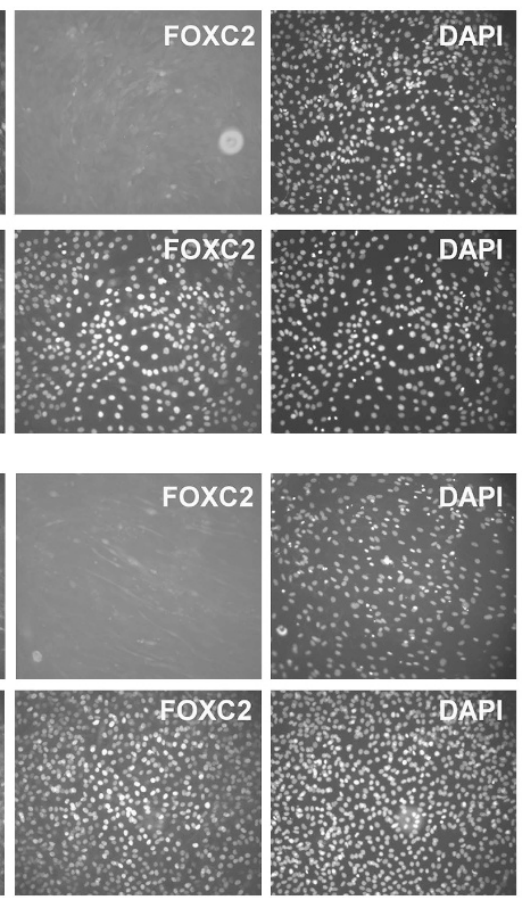

b
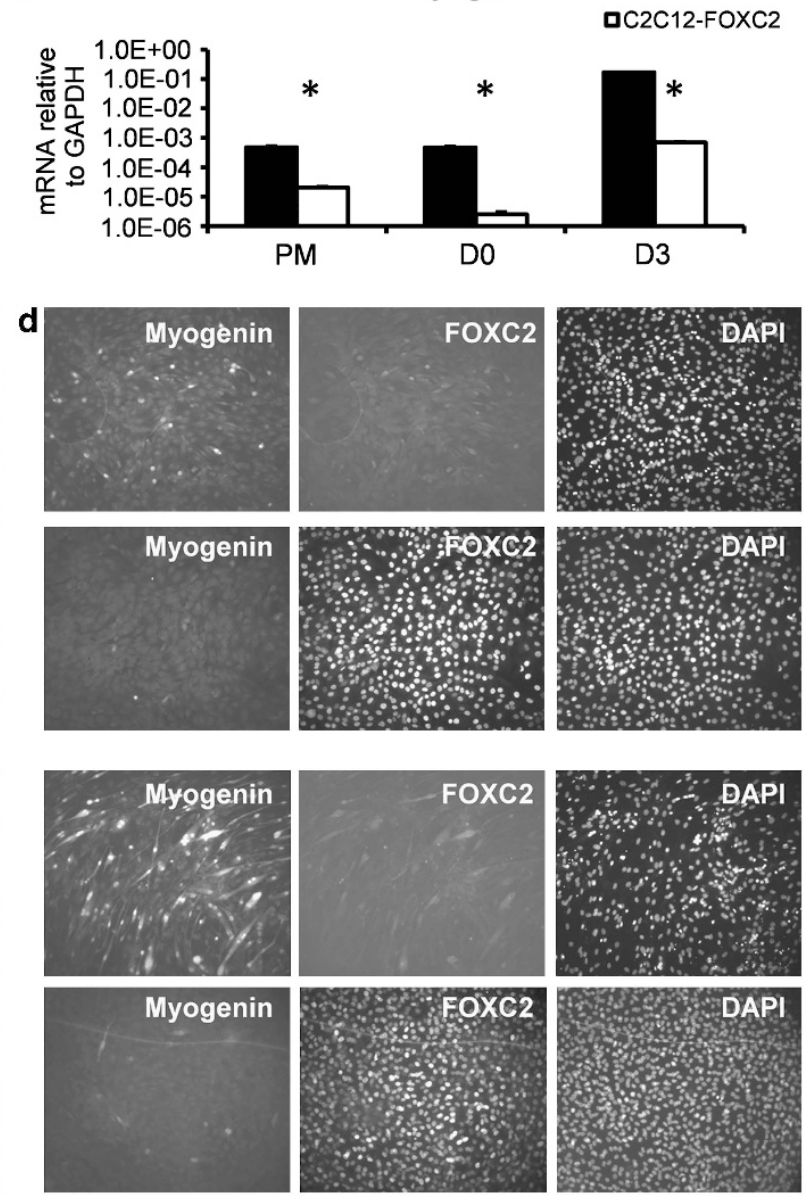

Figure 3 Markers of myogenic differentiation diminish upon forced continuous expression of FOXC2. (a and $\mathbf{b})$ qRT-PCR of the myogenic regulatory factors MyoD (a) and myogenin (b) was performed on mRNA isolated from C2C12-EV and C2C12-FOXC2 cells during the proliferation stage (PM), at 100\% confluency (D0) and 3 days post induction of myogenesis (D3). (c and d) Protein expression of $\mathrm{MyoD}$ (c) and myogenin (d) during myogenesis at D0 and D3 was assessed by immunofluorescence staining. Cells were visualized at $\times 10$ magnification using an Olympus IX51 fluorescence microscope. Images are representative of three independent experiments. ${ }^{*}$ represents statistically significance with $P<0.005$ between two groups

cells (PM and D0) and low in terminally differentiated muscle cells (D3) (Figures 3a and c). In comparison, C2C12-FOXC2 cells maintained moderate levels of MyoD throughout differentiation (Figures $3 \mathrm{a}$ and $\mathrm{c}$ ). Progression toward terminally differentiated myotubes involves upregulation of myogenin. Indeed, the levels of myogenin mRNA (Figure 3b) and protein (Figure $3 d$ ) were significantly lower in $\mathrm{C} 2 \mathrm{C} 12-$ FOXC2 than in C2C12-EV cells. These results indicate that downregulation of Foxc2 in undifferentiated proliferating myoblasts is a prerequisite for myogenesis.

Forced expression of FOXC2 in $\mathrm{C} 2 \mathrm{C} 12$ cells enhances myoblast proliferation, and delays cell-cycle withdrawal and apoptosis. Induction of differentiation directs activated satellite cells to undergo apoptosis or irreversibly withdraw from the cell cycle and commit toward the differentiation program. Comparison of expression profiles between C2C12-EV and C2C12-FOXC2 cells at 3 days post myogenic induction (D3) revealed an enrichment of several genes involved in cell-cycle progression in C2C12-FOXC2 cells (Supplementary Table S1D) and a cell proliferation assay demonstrated an enhanced rate of proliferation in $\mathrm{C} 2 \mathrm{C} 12-$ FOXC2 cells compared with C2C12-EV cells (Figure 4a). At $72 \mathrm{~h}$ post myogenic induction, proliferation had diminished in C2C12-EV cells whereas C2C12-FOXC2 cells continued to proliferate up to $120 \mathrm{~h}$ (Figure 4a). To determine whether expression of FOXC2 prevents cell-cycle withdrawal after myogenic induction, cells were subjected to cell-cycle analysis by FACS at different time points. A lower percentage of C2C12-FOXC2 cells underwent G1 arrest during myogenic induction compared with C2C12-EV cells (Figure 4b). This finding was confirmed by decreased p27 levels observed in C2C12-FOXC2 cells (Figure 4c). The slight decrease in G2 arrest that we observed in C2C12-FOXC2 cells (Figure 4b) was confirmed by suppressed phosphorylation of Cdc25C at serine-216 (Figure 4c). To determine whether FOXC2 prevents myoblasts from undergoing apoptosis when differentiation is induced, we analyzed $\mathrm{C} 2 \mathrm{C} 12$ myoblasts for early and late apoptosis by Annexin- $V$ staining. As expected, at $24 \mathrm{~h}$ post myogenic induction, the rate of apoptosis was increased in C2C12-EV cells and attenuated in C2C12-FOXC2 cells (Figure 4d). 
a

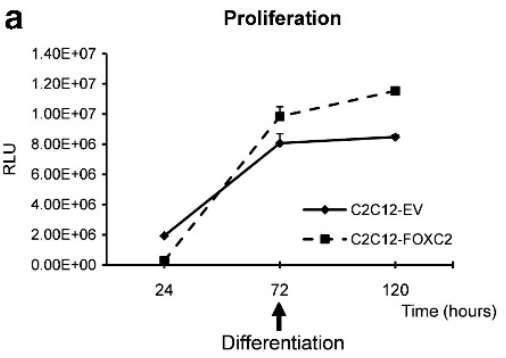

C

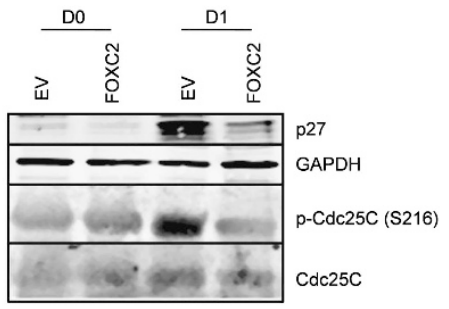

b

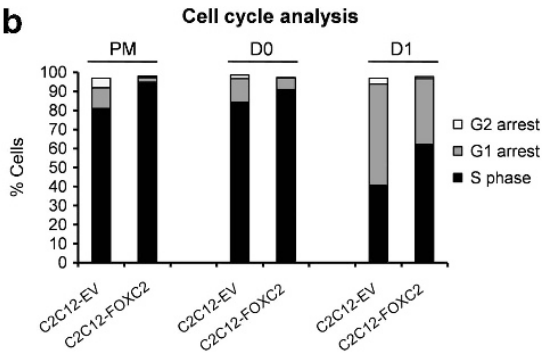

d

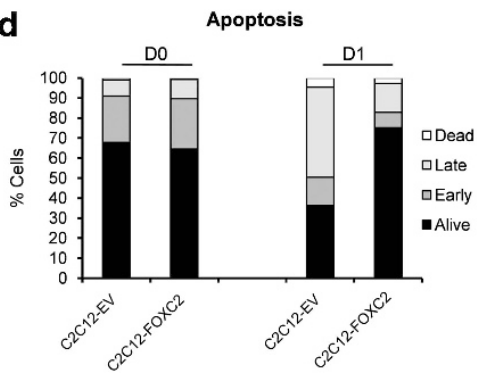

Figure 4 Forced expression of FOXC2 in $\mathrm{C} 2 \mathrm{C} 12$ cells enhances myoblast proliferation, and delays cell-cycle exit and apoptosis upon myogenic induction. (a) The rate of proliferation of C2C12-EV and C2C12-FOXC2 cells was measured using Celltiterglo at 24, 72 and $120 \mathrm{~h}$ post seeding. Differentiation was induced at $72 \mathrm{~h}$. (b) C2C12-EV and C2C12-FOXC2 cells were analyzed for cell-cycle progression by PI staining in undifferentiated proliferating myoblasts (PM), $100 \%$ confluent cells (D0) and in cells $24 \mathrm{~h}$ post myogenic induction (D1). The data are representative of three independent experiments. (c) C2C12-EV and C2C12-FOXC2 cells were harvested at D0 and D1 for protein expression analysis of the cell-cycle regulators p27 and Cdc25c by western blotting. GAPDH was used as a loading control. (d) The rate of apoptosis was analyzed by Annexin-V staining at the D0 and D1 time points. The data are representative of three or more independent experiments

To determine whether endogenous Foxc2 is important for proliferation of undifferentiated myoblasts, we generated C2C12 cell lines with stable Foxc2 knockdown (sh1 Foxc2 and sh2 Foxc2) or a scrambled shRNA control (Supplementary Figures S2A and $\mathrm{B}$ ). We found that downregulation of endogenous Foxc2 expression in $\mathrm{C} 2 \mathrm{C} 12$ myoblasts results in a significant decrease in cell proliferation and cell-cycle progression (Supplementary Figures S2C and D). Downregulation of Foxc2 in $\mathrm{C} 2 \mathrm{C} 12$ cells resulted in a delay in reaching $100 \%$ confluency, which is necessary for myogenic induction. When seeded at low confluency, sh1 Foxc2 and sh2 Foxc2 C2C12 cells required an additional $24 \mathrm{~h}$ of proliferation prior to myogenic induction in comparison with the scrambled cells (data not shown). However, when C2C12 myoblasts with downregulated Foxc2 were induced to undergo myogenic differentiation upon reaching 100\% confluency, we found that downregulation of Foxc2 had no significant effect in the later stages of myogenic differentiation as evidenced by efficient myotube formation and expression of myogenin (data not shown), which is consistent with the observed suppression of Foxc2 expression in the later stages of myogenic differentiation (Figures $2 b$ and $c$ ). We conclude that FOXC2 affects cell-cycle exit and cell survival during myogenic induction and maintains cells in their proliferative state.

FOXC2 regulates the transcription of Wnt4, resulting in the activation of both canonical and non-canonical Wnt pathways. To identify the downstream targets of Foxc2 that are involved in preventing myogenesis, we performed expression profile analysis in C2C12-EV and C2C12-FOXC2 cells, and compared the differences in gene expression at different stages of differentiation (PM and D3) (Supplementary Table S1). Comparison of expression profiles showed significantly higher levels of Wnt4 expression in C2C12-FOXC2 than in C2C12-EV cells at the PM (Supplementary Table S1B) and D0 (data not shown) time points. This finding was confirmed by qRT-PCR, which showed a significant upregulation of Wnt4 in C2C12-FOXC2 cells compared with $\mathrm{C} 2 \mathrm{C} 12-\mathrm{EV}$ cells for all four time points analyzed (Figure 5a). We identified a putative Foxc2 DNAbinding motif in an upstream promoter region of Wnt4. We were unable to determine whether endogenous mouse Foxc2 binds to the Wnt4 promoter region because our antimouse Foxc2 antibody was not effective in chromatin immunoprecipitation (ChIP). This could be due to the low level of Foxc2 expression at $24 \mathrm{~h}$ post myogenic induction. To determine whether ectopically expressed human FOXC2 associates with the Wnt4 promoter region, we performed ChIP-qPCR in C2C12-EV and C2C12-FOXC2 cells at D1 using antibodies against RNA polymerase-II (Pol-II) and human FOXC2. There was an enrichment of Pol-II and FOXC2 occupancy within the same Wnt4 promoter region in C2C12-FOXC2 cells compared with C2C12-EV cells (Figure $5 b$ ). Upregulation of Wnt4 has been reported to affect the activation of both canonical Wnt $\beta \mathrm{C}$ and noncanonical Wnt-planar cell polarity (PCP) signaling pathways. $^{20}$ To determine whether the canonical Wnt- $\beta \mathrm{C}$ pathway is activated by Foxc2, we analyzed myoblasts for TCF/LEF promoter activity using the TOPflash dual luciferase reporter assay at D1 and found a significant increase in TOPflash activity in C2C12-FOXC2 cells compared with C2C12-EV cells (Figure 5c). FOPflash, which contains a mutation in the TCF/LEF promoter that prevents 

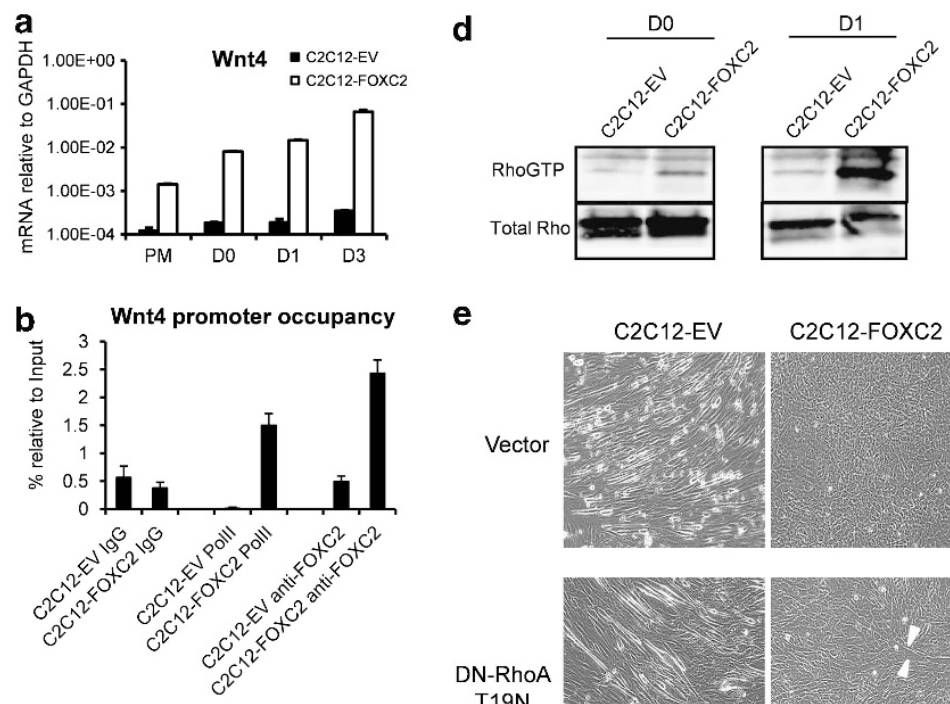

e
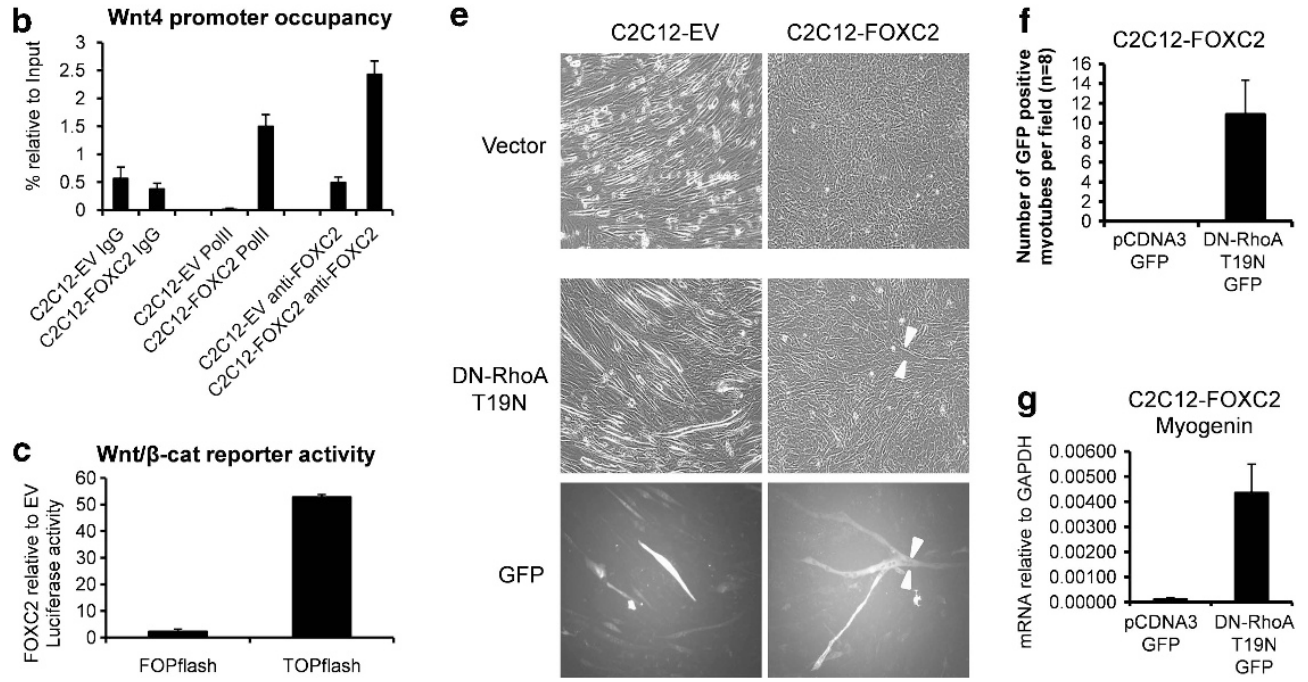

Figure 5 FOXC2 upregulates Wnt4 expression resulting in the activation of Wnt $/ \beta C$ and RhoGTP signaling pathways. (a) Wnt4 mRNA levels were analyzed by qRT-PCR in C2C12-EV and C2C12-FOXC2 cells at 75\% confluency (PM), 100\% confluency (D0), and at days 1 and 3 post myogenic differentiation. (b) ChIP using anti-Pol-II and antihuman FOXC2 antibodies was performed in C2C12-EV and C2C12-FOXC2 cells at D1 and analyzed for interaction of ectopically expressed FOXC2 with the Wnt4 promoter region containing the putative FOXC2 DNA-binding domain. (c) A TOPflash and FOPflash dual luciferase assays were performed in C2C12-EV and C2C12-FOXC2 cells at D1 to assess Wnt/ $\beta$ C signaling activity. (d) Expression levels of RhoGTP were analyzed in C2C12-EV and C2C12-FOXC2 cells at D0 and D1 by pull-down using Rhottekin RBD agarose that binds to active RhoA and western blot analysis using an anti-RhoA monoclonal antibody. (e) C2C12-EV and C2C12-FOXC2 cells were seeded at equal densities and transfected with a dominant-negative RhoA (RhoA T19N-GFP). The cells were analyzed with a fluorescence microscope for myotube formation 5 days post myogenic induction. Images were taken with a $\times 10$ objective and are representative of three independent experiments. (f) The number of multi-nucleated myotubes in C2C12-FOXC2 cells transfected with RhoA T19N-GFP or a control GFP vector was quantified and plotted to show the average number of myotubes per field $(n=8)$. (g) Expression of the myogenic differentiation marker myogenin was analyzed by qRT-PCR in C2C12-FOXC2 cells transfected with RhoA T19N-GFP or a control GFP vector

the $\beta \mathrm{C}-\mathrm{TCF}$ complex from binding to the region, served as the control (Figure 5c).

Previous studies have shown that Wnt-PCP signaling leads to Wnt-induced Dishevelled (Dvl)-Rho complex formation and activation of ras homolog family member-A (RhoA). ${ }^{21}$ We observed higher RhoGTP levels in C2C12-FOXC2 cells than in C2C12-EV cells (Figure $5 d$ ), suggesting that Wnt-PCP signaling may also be upregulated by FOXC2 in myogenic precursor cells. Previous studies have shown that downregulation of RhoGTP activity is required to initiate myogenesis in vitro. ${ }^{22,23}$ To determine whether suppression of RhoGTP activity in C2C12-FOXC2 cells rescues myotube formation, we transfected $\mathrm{C} 2 \mathrm{C} 12-\mathrm{EV}$ and $\mathrm{C} 2 \mathrm{C} 12-\mathrm{FOXC2}$ cells with a dominant-negative RhoA fused with GFP (DN-RhoA T19N) and induced myogenic differentiation. We analyzed the cells for myotube formation 5 days post induction and observed a significant increase in myotube formation in C2C12-FOXC2 cells that were transfected with the dominantnegative RhoA compared those transfected with the vector alone (Figures $5 e$ and f). In addition, we found that $\mathrm{C} 2 \mathrm{C} 12$ FOXC2 cells transfected with the dominant-negative RhoA had an increased expression of the myogenic differentiation marker myogenin compared with C2C12-FOXC2 cells transfected with the control GFP vector (Figure $5 \mathrm{~g}$ ). We conclude that the elevated levels of RhoGTP due to FOXC2 expression are involved in preventing myogenic differentiation.

To determine whether Wnt4 acts downstream from Foxc2 in preventing myogenic differentiation, we stably knocked down Wnt4 in C2C12-EV and C2C12-FOXC2 cells using two lentiviral shRNAs (Wnt4 sh1 and Wnt4 sh2), with a scrambled shRNA (scr) as a control (Figure 6a). C2C12-EV and C2C12FOXC2 cells with scr, Wnt4 sh1 and Wnt4 sh2 were then induced to undergo myogenesis in vitro. We observed an increase in cell alignment for myotube formation in the C2C12-FOXC2 Wnt4 sh2 cells compared with the C2C12FOXC2 scr cells (Figure 6b). Partial myotube formation, similar to that observed in the C2C12-FOXC2 Wnt4 sh2 cells, was also observed in the C2C12-FOXC2 Wnt 4 sh1 cells (data not shown). We then analyzed $\beta \mathrm{C}$ activity in C2C12-FOXC2 Wnt4-knockdown cells. We performed the TOPflash/FOPflash dual luciferase reporter assay to determine whether FOXC2-induced $\beta C$ activity can be suppressed by knockdown of Wnt4 expression. We observed a significant decrease in TCF/LEF promoter activity in the C2C12-FOXC2 Wnt4knockdown cells compared with the C2C12-FOXC2 scr 


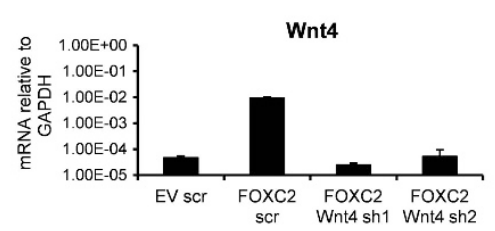

b
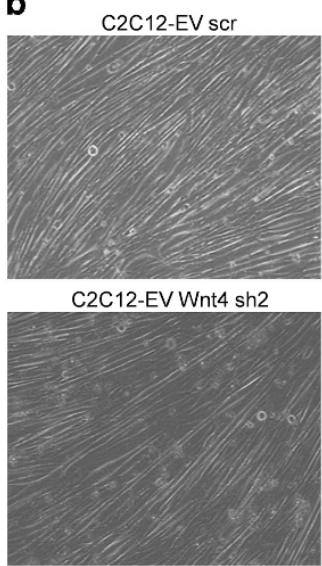

C2C12-FOXC2 scr

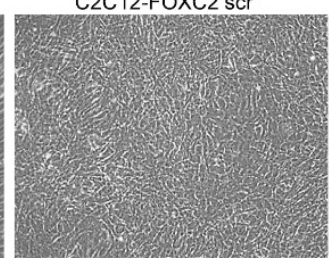

C2C12-FOXC2 Wnt4 sh2

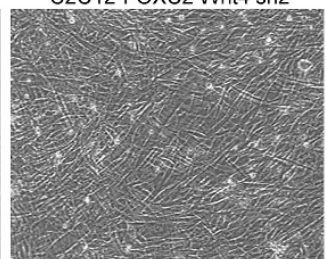

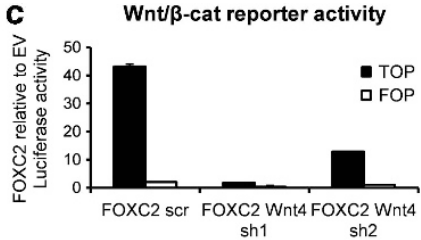

d

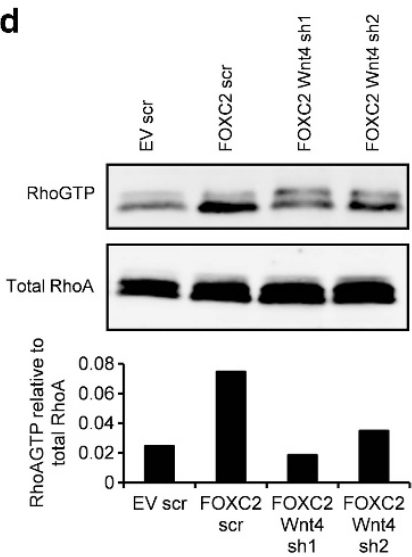

Figure 6 Wnt4 knockdown in C2C12-FOXC2 cells results in partial rescue of myotube formation, and a decrease in Wnt/ $\beta$ C signaling and RhoGTPase activity. (a) C2C12FOXC2 cell lines with stable Wnt4 knockdown were generated using two lentiviral Wnt4 shRNAs (sh1 and sh2). Wnt4 knockdown was confirmed by qRT-PCR. (b) C2C12-EV and C2C12-FOXC2 cells with a scrambled shRNA (scr) or an shRNA targeting Wnt4 (Wnt4 sh2) were induced to undergo myogenic differentiation. The cells were analyzed for myotube formation 5 days post induction. (c) A TOPflash and FOPflash dual luciferase reporter assay was performed in C2C12-EV and C2C12-FOXC2 cells with Wnt4 knockdown or the scrambled control to determine Wnt $/ \beta C$ activity $24 \mathrm{~h}$ post myogenic induction (D1). The cells were analyzed for luciferase activity $48 \mathrm{~h}$ post shRNA transfection. The fold changes between C2C12-FOXC2 scr and Wnt4-knockdown reporter activities were compared relative to the control C2C12-EV scr reporter activity. (d) The cells were analyzed for RhoGTPase activity at D1 by pull-down using Rhottekin beads and western blot analysis using an anti-RhoA monoclonal antibody. The lower panel shows the densitometry values of the western blot that was scanned with an Odyssey infrared imaging system and quantified using the Image Studio software

controls (Figure 6c). We also analyzed RhoGTP levels in the C2C12-EV scr, C2C12-FOXC2 scr and C2C12-FOXC2 Wnt4knockdown cells, and observed a decrease in RhoGTP levels in the cell lines with Wnt4 knockdown (Figure 6d). These results show that FOXC2 participates in regulating Wnt- $\beta \mathrm{C}$ signaling and RhoGTPase activity through upregulation of Wnt4 expression, which in turn inhibits myogenesis.

Forced expression of FOXC2 in $\mathrm{C} 2 \mathrm{C} 12$ cells leads to osteogenic induction by upregulation of Bmp4. Histologic sections of muscle samples from patients diagnosed with myositis ossificans showed positive nuclear staining for FOXC2 in areas of ectopic bone formation (Figure 7a), suggesting that upregulation of FOXC2 may play a role in ectopic bone development in patients with heterotopic ossification. A normal condition medium containing fetal bovine serum (FBS) is rich in growth factors and contains BMP4. Under these conditions, C2C12 myoblasts maintain their proliferative undifferentiated state and retain their capability to differentiate toward the myogenic lineage upon myogenic induction. However, with addition of high levels of exogenous BMP2 and continuous activation of BMPR-1A signaling, $\mathrm{C} 2 \mathrm{C} 12$ myoblasts can be induced to differentiate toward the osteogenic lineage in vitro. ${ }^{7}$ To determine whether continuous overexpression of FOXC2 enhances the transdifferentiation of $\mathrm{C} 2 \mathrm{C} 12$ cells toward osteogenesis, we induced C2C12-EV and C2C12-FOXC2 cells to undergo osteoblast differentiation using recombinant BMP2. After 1, 2 and 5 days, we stained formalin-fixed cells for alkaline phosphatase (ALP), a marker of osteogenesis, and found that FOXC2 overexpression had dramatically enhanced osteogenesis. We observed positive ALP staining as early as 1 day post osteogenic induction in C2C12-FOXC2 cells (Figure 7b). Osteogenic induction performed in NIH3T3 fibroblasts yielded similar results, with enhanced BMP2induced ALP activity observed in NIH3T3-FOXC2 cells compared with NIH3T3-EV cells (Supplementary Figure S3).

Interestingly, we observed moderate ALP staining at day 5 in C2C12-FOXC2 cells (Figure 7b) and at day 14 in NIH3T3FOXC2 cells (Supplementary Figure S3) even without addition of BMP2. Expression profile analysis of C2C12FOXC2 cells relative to C2C12-EV cells also identified an upregulation of genes involved in bone formation in the absence of BMP2 (Supplementary Table S1). Using qRT$\mathrm{PCR}$, we confirmed that there was an increase in the mRNA levels of the osteogenic markers Runt-related transcription factor-2 (Runx2), Osterix and osteocalcin in C2C12-FOXC2 cells compared with $\mathrm{C} 2 \mathrm{C} 12-\mathrm{EV}$ cells (Figure 7c), indicating that FOXC2 may direct $\mathrm{C} 2 \mathrm{C} 12$ myoblasts toward osteogenic differentiation by elevating endogenous expression of BMPs. Indeed, expression profile analysis of proliferating myoblasts detected an upregulation of Bmp4 in C2C12-FOXC2 cells compared with C2C12-EV cells (Supplementary Table S1B). These results were confirmed by qRT-PCR and western blot 
a

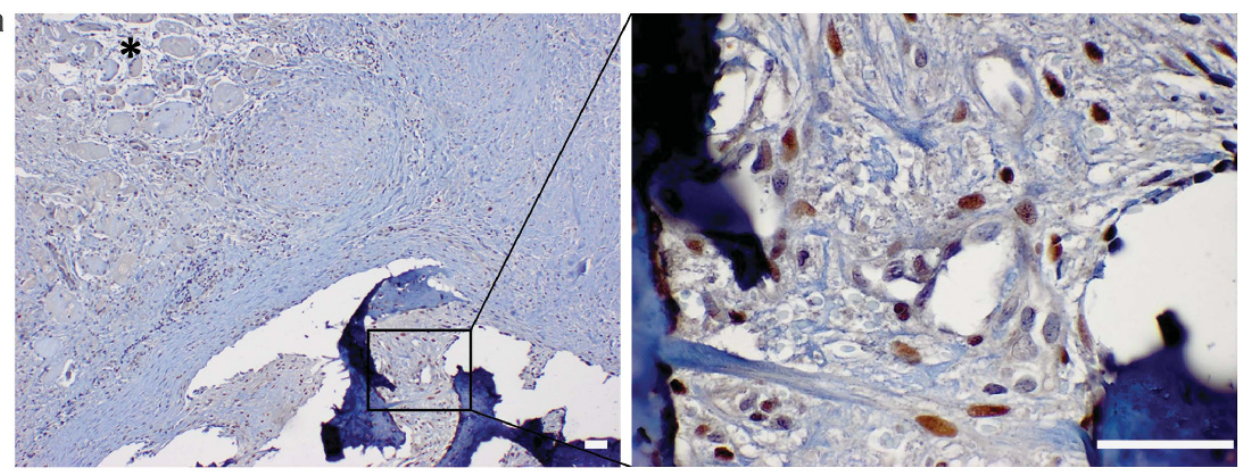

b

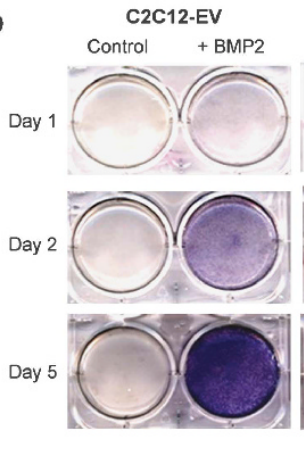

C

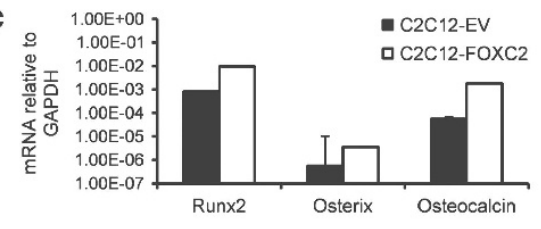

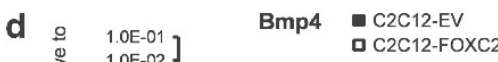
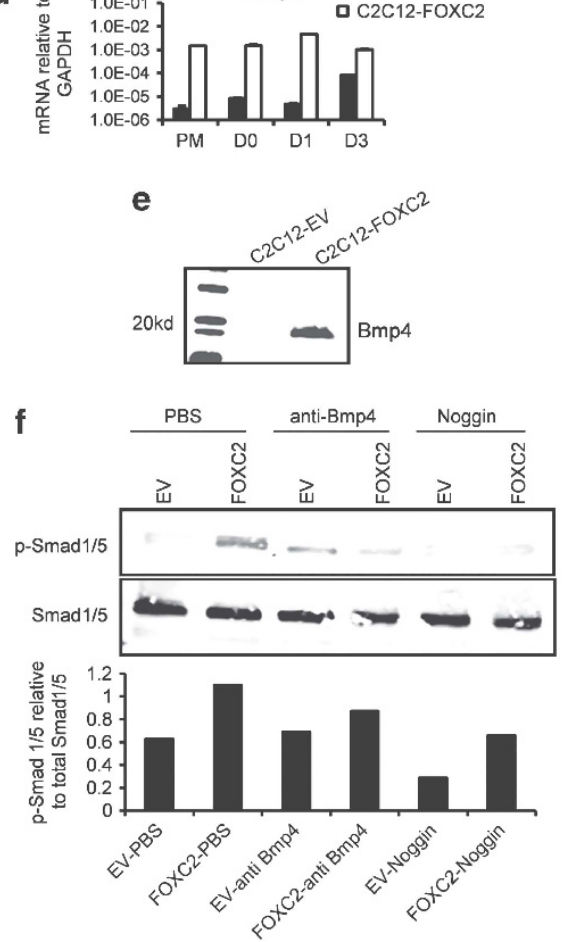

g

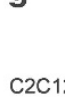

EV
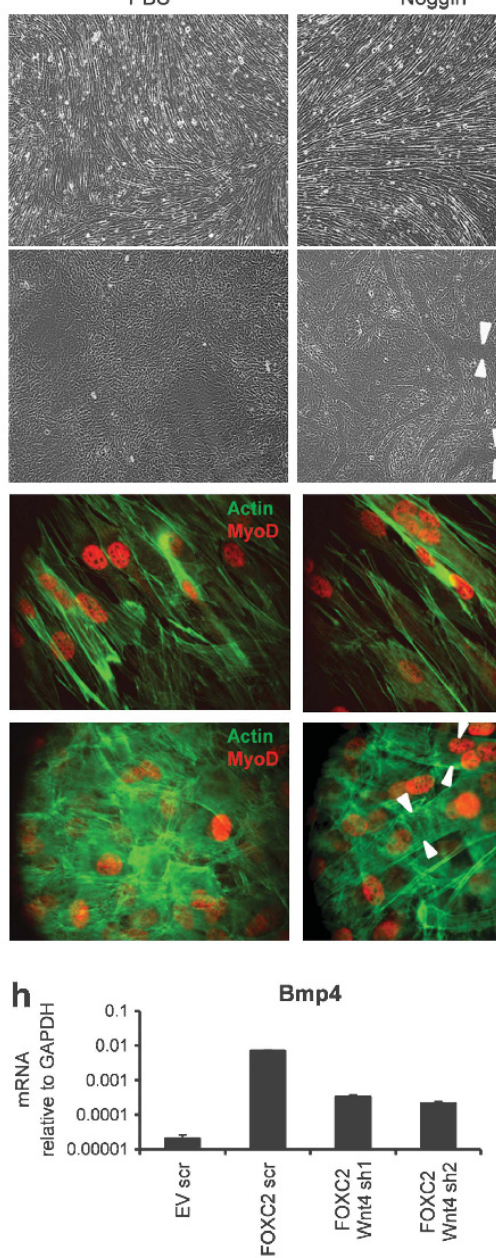

i

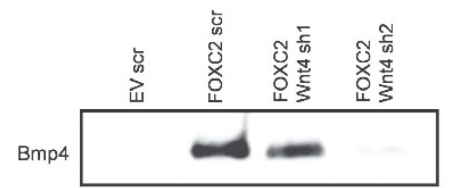


analysis of Bmp4 mRNA and secreted Bmp4 protein, respectively (Figures $7 d$ and $e$ ). Bmp4 has been shown to activate Bmpr1a, which leads to phosphorylation of Smad1/ $5^{24}$ Indeed, increased phosphorylation of Smad1/5 was detected in C2C12-FOXC2 cells compared with C2C12-EV cells (Figure $7 f$ ). In the presence of a neutralizing antibody against Bmp4 or in the presence of the Bmp4 antagonist Noggin, we observed decreased Smad1/5 phosphorylation in C2C12-FOXC2 cells compared with cells without Bmp4 inhibitors (Figure 7f). To determine whether inhibition of Bmp4 signaling in C2C12-FOXC2 cells can rescue myotube formation, we induced myogenesis in C2C12-EV and C2C12FOXC2 cells in the presence or absence of Noggin, and assessed myotube formation by bright-field microscopy and fluorescence microscopy. We observed partial rescue of myotube formation in C2C12-FOXC2 cells in the presence of Noggin (Figure 7g). Collectively, these results show that forced expression of $\mathrm{FOXC2}$ in $\mathrm{C} 2 \mathrm{C} 12$ cells results in upregulation of Bmp4, which can re-direct the differentiation of $\mathrm{C} 2 \mathrm{C} 12$ myoblasts from myogenesis toward osteogenesis. We additionally show that in undifferentiated C2C12 myoblasts, a decrease in endogenous Foxc2 levels results in a decrease in Bmp4 mRNA expression (Supplementary Figure S2E), suggesting that moderate levels of Bmp4 may be important for the maintenance of the proliferative state of undifferentiated myoblasts. We then asked if the upregulation of Bmp4 in C2C12-FOXC2 cells was downstream from elevated Wnt4 signaling. The Wnt4 knockdown resulted in a significant decrease in Bmp4 expression at both the mRNA and protein levels (Figures $7 \mathrm{~h}$ and i). We conclude that FOXC2-induced Wnt4 signaling produces elevated levels of RhoGTP and Bmp4, which results in osteogenic differentiation in $\mathrm{C} 2 \mathrm{C} 12$ cells.

\section{Discussion}

Muscle regeneration is a complex process that requires tight regulation and coordination of several signaling molecules. ${ }^{25-27}$ Genes involved in muscle satellite cell proliferation and muscle differentiation are tightly regulated and expressed at specific time points during the regenerative process. $^{28}$ Although it is known that activation of the transcription factors Smad and Tcf through the Bmp (Smad) and Wnt (Tcf) signaling pathways is essential during the temporal switch from satellite cell proliferation to differentiation in muscle tissue, ${ }^{29}$ the molecular mechanism that regulates and coordinates these pathways during muscle regeneration is poorly understood. In this study, we identified a novel mechanism whereby Foxc2 plays a role in enhancing myoblast proliferation and preventing myogenic differentiation by regulating both the Wnt and Bmp signaling pathways.

We show that during adult muscle regeneration, Foxc2 is expressed in $\mathrm{Pax}^{+} / \mathrm{MyoD}^{+}$activated muscle satellite cells, which are thought to be multipotent and the source of myonuclei in regenerating muscle. We found that ectopically expressed FOXC2 interacts with the upstream promoter region of Wnt4 and that this interaction induces Wnt4 expression, resulting in increased $\mathrm{Wnt} / \beta \mathrm{C}$ signaling and elevated RhoGTP levels. We demonstrated that knockdown of Wnt4 in C2C12-FOXC2 cells results in downregulation of Wnt/ $\beta$ C signaling, RhoGTP levels and Bmp4 expression. Both Wnt and Bmp signaling have been reported to act synergistically in regulating cell-specific programs during differentiation and regeneration. ${ }^{30,31}$ It was shown that Wnts and BMPs can cooperatively induce early osteoblast differentiation in C2C12 cells in a GSK3 $\beta$-dependent manner, ${ }^{32}$ and that constitutive activation of RhoA in human mesenchymal stem cells can induce osteogenesis in vitro. ${ }^{33}$ However, it has not been determined whether upregulation of both Wnt and Bmp signaling produces an additive induction of RhoGTP expression. We were unable to determine whether downregulation of Bmp4 signaling alone had any effect on the enhanced RhoGTP expression in C2C12-FOXC2 cells. While it is possible that induction of osteoblast differentiation is the result of a synergistic upregulation of RhoA-GTP levels by both Wnt and Bmp signaling, further studies are needed to determine if this is the case.

Bmp4 has been shown to play a role in muscle satellite cell proliferation and survival. ${ }^{7}$ Although expression of Bmp4 prevents premature myogenesis, continuous expression of Bmp4 in mesenchymal cells primed to undergo differentiation was shown to induce osteogenesis or chondrogenesis. ${ }^{31,34}$ FOXC2-induced Bmp4 expression altered the commitment of these myogenic precursor cells toward osteogenesis as demonstrated by an increase in ALP staining and upregulation of osteogenic markers. Several groups have reported that downregulation of Bmp signaling is important in regulating the expansion of muscle precursor cells and in preventing myogenic differentiation. ${ }^{7,10,35}$ Consistent with these reports, we show that the presence of the Bmp4 antagonist Noggin results in partial rescue of myotube fusion in C2C12-FOXC2 cells.

Figure 7 FOXC2 alters the lineage commitment of myoblasts toward osteogenesis by upregulation of Bmp4. (a) A representative image of a heterotopic bone in a muscle of a patient with myositis ossificans. Sections of formalin-fixed, paraffin-embedded tissue biopsies were stained using a FOXC2 antibody. The asterisk indicates the muscle area. The heterotopic bone area in the rectangle is shown at high magnification. Images were taken with an Olympus DP25 camera with an Olympus BX43 light microscope. Scale bar, $50 \mu \mathrm{m}$. (b) C2C12-EV and C2C12-FOXC2 cells were induced to undergo osteogenesis using recombinant BMP2. The cells were stained for bone ALP at days 1,2 and 5 post induction. (c) Expression levels of the osteogenic markers Runx2, Osterix and osteocalcin in C2C12-EV and C2C12-FOXC2 cells in the absence of BMP2 at 5 days post confluency were analyzed by qRT-PCR. (d) Analysis of Bmp4 mRNA expression by qRT-PCR in C2C12-EV and C2C12-FOXC2 cells during myogenic induction. (e) Western blot analysis of secreted Bmp4 in cell culture media of $\mathrm{C} 2 \mathrm{C} 12-\mathrm{EV}$ and $\mathrm{C} 2 \mathrm{C} 12-\mathrm{FOXC} 2$ cells at day 1 post myogenic induction. (f) Western blot analysis of phosphoSmad1/5 in C2C12-EV and C2C12-FOXC2 cells in the presence of PBS, a Bmp4-neutralizing antibody (anti-Bmp4) or the Bmp4 inhibitor Noggin. The lower panel shows the densitometry values of the western blot that was scanned with an Odyssey infrared imaging system and quantified using the Image Studio software. (g) Myogenic induction of $\mathrm{C} 2 \mathrm{C} 12-\mathrm{EV}$ and C2C12-FOXC2 cells was performed in the presence of PBS or Noggin, and analyzed for myotube formation at day 5 post myogenic induction by bright-field microscopy (upper panels) and immunofluorescence visualization of cytoskeletal actin and nuclear MyoD (lower panels). The white arrowheads indicate partial myotube formation in C2C12-FOXC2 cells. ( $h$ and i) Bmp4 expression in C2C12-FOXC2 Wnt4-knockdown cells was analyzed for Bmp4 mRNA by qRT-PCR (h) and for secreted protein expression by western blot analysis using an antibody against Bmp4 (i) 
Dysregulation of Bmp4 signaling and/or its receptor has been implicated in heterotopic ossification and myositis ossificans, ${ }^{36,37}$ and several studies have suggested that Bmp4 plays a key role in the development of these diseases. ${ }^{36,38,39}$ Heterotopic ossification was recapitulated in an animal model by inducing muscle damage that resulted in the recruitment and activation of immune cells, endothelial cells and mesenchymal progenitors, and an increase in myoblasts in muscle tissue. Activated $\mathrm{Bmp}$ signaling initiated endochondral ossification at the site of muscle injury. ${ }^{40}$ Although it is still unclear if FOXC2 plays a role in the development of conditions such as heterotopic ossification, we show that FOXC2 is expressed in areas of ectopic bone development in muscle biopsies from patients diagnosed with myositis ossificans.

In conclusion, we propose that the transcription factor Foxc2 plays a role in maintaining the balance between the proliferative and undifferentiated state of myogenic precursor cells. Foxc2 acts as a modulator of both the Wnt and Bmp signaling pathways (Figure 8). To our knowledge, this is the first demonstration of the expression and function of Foxc2 in muscle satellite cells. Further characterization of Foxc2 regulation will help elucidate the mechanisms involved in cell fate determination during muscle regeneration and provide greater insight into the pathology of heterotopic ossification.

\section{Materials and Methods}

Cell lines. Mouse $\mathrm{C} 2 \mathrm{C} 12$ myoblasts and $\mathrm{NIH} 3 \mathrm{~T} 3$ fibroblasts were obtained from American Type Culture Collection (ATCC, Manassas, VA, USA). Proliferating undifferentiated myoblasts were maintained at subconfluent density in high-serum growth medium (DMEM, 15\% FBS and 1\% penicillin/streptomycin (PS)). NIH3T3 fibroblasts were maintained in standard growth medium (DMEM, 10\% FBS and $1 \%$ PS).

Retroviral infection. The retroviral vectors pWZL-blast and pWZL-FOXC2 (provided by Mani Sendurai) were transfected into LinXA cells seeded in a six-well plate at $4 \times 10^{5} \mathrm{cells} /$ well using Lipofectamine 2000 (Invitrogen, Grand Island, NY, USA). After $72 \mathrm{~h}$, the viral supernatant was harvested into a $50-\mathrm{ml}$ conical tube using a $0.45-\mu \mathrm{m}$ filter syringe. Approximately $250 \mu \mathrm{l}$ of the viral supernatant was used to transduce $\mathrm{C} 2 \mathrm{C} 12$ cells that had been seeded the previous day at $3 \times 10^{4}$ cells/well in a six-well plate. High-serum growth medium with $8 \mu \mathrm{g} / \mathrm{ml}$

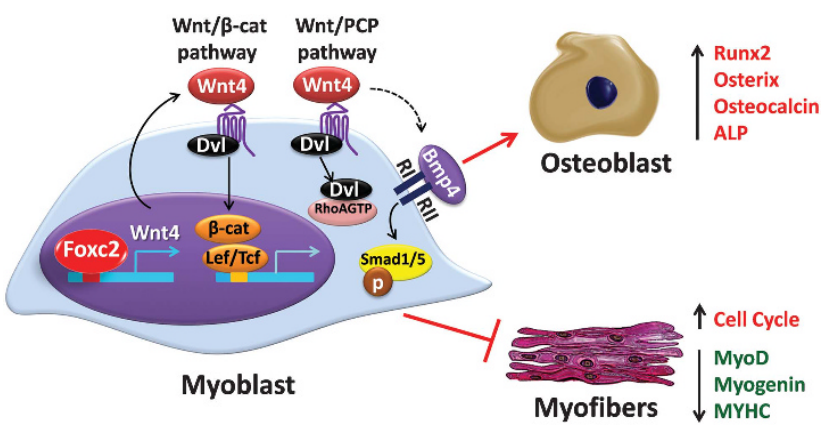

Figure 8 Proposed model for FOXC2 regulation of myoblast proliferation and osteogenic differentiation via upregulation of the Wnt and Bmp signaling pathways. FOXC2 is present in mesenchymal progenitor cells, but its expression diminishes during myogenic differentiation. Forced continuous expression of FOXC2 in $\mathrm{C} 2 \mathrm{C} 12$ myoblasts promotes the binding of FOXC2 to the promoter region of Wnt4, which in turn leads to upregulation of Wnt4 expression and activation of canonical $(\beta C)$ and non-canonical (RhoA) Wnt signaling, as well as increased levels of RhoGTP and Bmp4. Failure to downregulate FOXC2 in muscle progenitors inhibits myogenesis and induces osteogenesis polybrene (Millipore, Billerca, MA, USA) was added to the $\mathrm{C} 2 \mathrm{C} 12$ cells prior to viral infection. After $24 \mathrm{~h}, 10 \mu \mathrm{g} / \mathrm{ml}$ blasticidin (Invivogen, San Diego, CA, USA) was added for the next $48 \mathrm{~h}$ to select a polyclonal population of blasticidin-resistant cells.

Wnt4 and Foxc2 knockdown using shRNA. pLKO.1-Wnt4 sh1 and sh2, pLKO.1-Foxc2 sh1 and sh2, and pLKO.1 scrambled shRNA plasmids (Sigma, St. Louis, MO, USA) were co-transfected with the delta 8.9 packaging plasmid and the envelope plasmid VSVG into Lenti-X 293T cells using Lipofectamine 2000 (Invitrogen). The harvesting procedure was identical to that used for the production of retroviruses. The viral supernatant was used for infection of cells with $8 \mu \mathrm{g} / \mathrm{ml}$ polybrene. The cells were infected overnight and polyclonal populations of stable infectants were selected for $48 \mathrm{~h}$ using $5 \mu \mathrm{g} / \mathrm{ml}$ puromycin.

Induction of myogenesis and osteogenesis. Myoblasts were maintained in high-serum growth medium (DMEM, 15\% FBS and 1\% PS) at less than $75 \%$ confluency. To induce differentiation, the cells were grown to $100 \%$ confluency and incubated in a differentiation medium (DMEM, 2\% FBS and 1\% PS). They were observed for morphological changes up to 7 days post myogenic induction. To inhibit Bmp signaling and induce myoblast fusion in C2C12-FOXC2 cells, $0.2 \mu \mathrm{g} / \mathrm{ml}$ human recombinant Noggin (R\&D Systems, Minneapolis, MN, USA) was added to the myogenic induction medium. Osteogenic induction of C2C12-EV and C2C12-FOXC2 cells was performed as described previously. ${ }^{41}$ Cells were plated at a density of $2 \times 10^{4} \mathrm{cells} / \mathrm{cm}^{2}$. After $24 \mathrm{~h}$, the medium was replaced with DMEM containing $5 \%$ FBS and $300 \mathrm{ng} / \mathrm{ml}$ human recombinant BMP2 (GenWay Biotech, San Diego, CA, USA) for the remainder of the experiment. After 5 days, osteoblast differentiation was analyzed by ALP staining (Sigma) using the protocol provided by the manufacturer.

Quantitative real-time PCR. RNA was isolated from cells at several time points during differentiation using the RNeasy kit (Qiagen, Valencia, CA, USA). For qRT-PCR, $50 \mathrm{ng}$ of $\mathrm{CDNA}$ was mixed with the appropriate primers and the iQSYBR-Green Supermix (Bio-Rad, Hercules, CA, USA) in a 96-well plate format. The qRT-PCR reaction was performed using an iCycler optical module and a thermocycler (Bio-Rad). Samples were run in triplicates and normalized to GAPDH or $18 \mathrm{~S}$ ribosomal RNA. The primer sequences used to detect myogenic and osteogenic markers are listed in Supplementary Table S2.

Western blot analysis. Whole-cell lysates were prepared using RIPA buffer (Sigma) containing a protease inhibitor cocktail (Roche, Indianapolis, IN, USA) and a phosphatase inhibitor cocktail (Roche). Proteins $(50 \mu \mathrm{g})$ were separated using $4-20 \%$ gradient Tris- $\mathrm{HCl}$ gel (Bio-Rad); transferred onto a nitrocellulose membrane (Bio-Rad); and probed for anti-human or anti-mouse FOXC2 antibody (N. Muira), and the myogenic markers MyoD, myogenin and myosin heavy chain (Santa Cruz Biotechnology, Santa Cruz, CA, USA). To detect the expression of secreted proteins, $8 \times 10^{4}$ cells were seeded in a $10-\mathrm{cm}^{2}$ dish and allowed to grow to $100 \%$ confluency. The cells were washed with PBS and incubated overnight in DMEM without serum. The following day, $10 \mathrm{ml}$ of medium was harvested from C2C12-EV and C2C12-FOXC2 cells, and concentrated to $200 \mu \mathrm{l}$ using $15-\mathrm{ml}$ centrifugal filters for collection of proteins larger than $10 \mathrm{kDa}$ (Millipore). Mouse monoclonal antibodies against Bmp4 (Millipore) and rabbit anti-Wnt4 (Santa Cruz Biotechnology) were used to detect the levels of the secreted proteins. Western blots were scanned and analyzed using the Li-Cor Odyssey infrared imaging system.

Immunofluorescence staining. Myoblasts were seeded at $3 \times 10^{4} \mathrm{cells} /$ well in a six-well plate containing $12 \times 12-\mathrm{mm}$ microscope coverslips. Proliferating cells were rinsed with PBS and fixed with $3 \%$ paraformaldehyde. The fixed cells were incubated with $0.1 \%$ Triton X-100 (Sigma) in PBS and stained for FOXC2 (N. Miura), and the muscle satellite cell markers Pax7 (Abcam, Cambridge, MA, USA), MyoD (Santa Cruz Biotechnology) and myogenin (Santa Cruz Biotechnology).

TOPflash and FOPflash dual luciferase assay. C2C12-EV and C2C12-FOXC2 cells were seeded in a six-well dish at $3 \times 10^{4}$ cells/well with growth medium (DMEM, 10\% FBS and $1 \%$ PS). After $24 \mathrm{~h}$, the cells were transfected with a luciferase reporter plasmid regulated by the TCF/LEF promoter TOPflash (Addgene plasmid 12456) or the mutated version of the promoter 
FOPflash (Addgene plasmid 12457) both deposited by Randall Moon. ${ }^{42}$ The cells were co-transfected with a plasmid expressing Renilla luciferase (Promega, Madison, WI, USA) using Lipofectamine 2000 (Invitrogen) following the manufacturer's suggested transfection protocol. The cells were analyzed for luciferase activity $48 \mathrm{~h}$ post transfection using a dual luciferase reporter assay system (Promega) and a microplate luminometer (Veritas).

Proliferation, cell-cycle and apoptosis assays. For cell proliferation analysis, myoblasts were seeded at $1 \times 10^{3}$ cells/well in 96-well plates. Celltiterglo (Promega) was used to detect viable proliferating cells following the manufacturer's protocol. Cells were detected using a luminometer (Veritas) and analyzed at three different time points during myogenic induction. To perform cellcycle analysis, $8 \times 10^{4}$ cells were seeded in a $10-\mathrm{cm}^{2}$ dish. Prior to cell harvesting, $15 \mu \mathrm{l}$ of BrdU (Amersham, Pittsburgh, PA, USA) was added to the cell culture overnight. The following day, cells were trypsinized, resuspended in $0.5 \mathrm{ml}$ of icecold PBS and fixed with $95 \%$ ethanol. The cells were stained with propidium iodide (PI) and incubated for $1 \mathrm{~h}$ at $37^{\circ} \mathrm{C}$, and then analyzed by FACS using a FACScan (BD Biosciences, San Jose, CA, USA). To detect cellular apoptosis using the phycoerythrin (PE) Annexin- $V$ apoptosis kit (BD Biosciences), cells were seeded as described for cell-cycle analysis and stained with PE Annexin-V and 7-aminoactinomycin-D (7-AAD) following the manufacturer's protocol. Cells were analyzed by FACS using a FACScan (BD Biosciences).

Measuring RhoGTP levels. Whole-cell lysates from C2C12-EV and C2C12-FOXC2 myoblasts were grown under pre-myogenic conditions followed by $24 \mathrm{~h}$ of post-myogenic conditions. The RhoA Assay Reagent (Upstate, Billerca MA, USA) was used to determine RhoGTP levels following the protocol provided by the manufacturer. Monoclonal mouse anti-RhoA (NewEast, King of Prussia, PA, USA) was used to detect RhoA by western blot analysis.

Transfection with dominant-negative RhoA. C2C12-EV and $\mathrm{C} 2 \mathrm{C} 12-$ FOXC2 cells were seeded at $3 \times 10^{4}$ cells in a six-well plate. The cells were transfected with a plasmid expressing a dominant-negative RhoA-GFP fusion protein (pCDNA3-EGFP-RhoA T19N). The plasmid was obtained from Addgene (Cambridge, MA, USA) (Addgene plasmid 12967), which was deposited by Gary Bokoch. Transfection with $4 \mu \mathrm{g}$ of the plasmid DNA was performed using the BioT transfection reagent (Bioland Scientific, Paramount, CA, USA) following the protocol provided by the manufacturer.

CTX and BrdU injection. All animal procedures were performed in accordance with the NIH Guide for the Care and Use of Laboratory Animals and approved by the Institutional Animal Care and Use Committee (IACUC) of Cedars-Sinai Medical Center. C57/BL6 mice aged 8-12 weeks were purchased from the Jackson Laboratories (Bar Harbor, PA, USA). To induce muscle injury, a $271 \frac{1}{2}$-gauge needle was used to inject $25 \mu$ l of PBS or a $10-\mathrm{mM}$ solution of Najamossambica CTX in PBS into the anterior tibialis and rectus femoris muscles. Prior to injection, mice were anesthetized with isoflurane inhalation. To analyze muscle satellite cell activation and proliferation, the mice were injected intraperitoneally with $250 \mu \mathrm{l}$ of $4 \mathrm{mg} / \mathrm{ml} \mathrm{BrdU}$ (GE Healthcare, Pittsburgh, PA, USA) $24 \mathrm{~h}$ prior to being killed at specific time points (day 4, day 6 and day 14). Muscle harvested from the injection sites was either fixed in formalin for immunohistochemical staining or prepared into a single-cell suspension for FACS analysis.

FACS analysis. Single-cell suspensions were prepared by harvesting mouse muscle and incubating it for $30 \mathrm{~min}$ at $37^{\circ} \mathrm{C}$ in PBS containing dispase $(2.5 \mathrm{U} / \mathrm{ml})$ and collagenase $\mathrm{XI}(0.2 \% \mathrm{wt} / \mathrm{vol})$. The cells were triturated and resuspended using a $70-\mu \mathrm{m}$ filter in a $50-\mathrm{ml}$ conical tube over ice and washed three times with icecold PBS. The cells were fixed in fixation/permeabilization buffer (eBioscience, San Diego, CA, USA) according to the manufacturer's protocol. A total of $1 \times 10^{6}$ cells were stained with the following primary antibodies: mouse anti-BrdU (BD Biosciences), mouse or rabbit anti-MyoD (Santa Cruz Biotechnology), rabbit antiPax7 (Abcam), rat anti-mouse Foxc2 (N. Miura) or isotype controls, followed by a secondary fluorescent antibody against primary antibody isotypes (Invitrogen). The cells were washed three times with permeabilization buffer (eBioscience) and analyzed using an LSRII flow cytometer (BD Biosciences).

Immunohistochemistry. Immunohistochemical studies involving human tissue samples were approved by the Cedars-Sinai Medical Center Review
Board. Formalin-fixed, paraffin-embedded blocks were retrieved from surgical pathology files at Cedars-Sinai Medical Center. Tissue sections were stained with a mouse anti-human FOXC2 antibody (N. Miura) at 1:100 dilution using the protocol described in our previous publication. ${ }^{43}$

Statistical analysis. qRT-PCR mean differences between groups were examined by Student's $t$-test (two-tailed) using commercially available software (GraphPad). $P<0.05$ was considered significant. Error bars in all graphs represent standard deviation in triplicate samples and asterisk indicates a significant difference by Student's $t$-test with a $P$-value $\leq 0.01$. FOXC2-induced differentially expressed genes were functionally annotated using the Gene Ontology and KEGG pathway (Kyoto Encyclopedia of Genes and Genomes), based on gene set enrichment analysis using DAVID (Database for Annotation, Visualization and Integrated Discovery). ${ }^{44}$

\section{Conflict of Interest}

The authors declare no conflict of interest.

Acknowledgements. We express our gratitude to the members of the Orsulic laboratory and Women's Cancer Program for helpful suggestions; Vincent Funari for assistance with expression profiling; Mani Sendurai for FOXC2 plasmid constructs; Beatrice Knudsen, Neil Bhowmick and Leland Chung for critical reading of the manuscript; and Kristy Daniels for assistance with manuscript preparation. S.O. is supported by grants from the American Cancer Society (RSG-10-252-01-TBG) and the Sarcoma Foundation of America, and funds from the Women's Cancer Program at Cedars-Sinai Medical Center.

1. Allbrook DB, Han MF, Hellmuth AE. Population of muscle satellite cells in relation to age and mitotic activity. Pathology 1971; 3: 223-243

2. Zammit PS, Heslop L, Hudon V, Rosenblatt JD, Tajbakhsh S, Buckingham ME et al. Kinetics of myoblast proliferation show that resident satellite cells are competent to fully regenerate skeletal muscle fibers. Exp Cell Res 2002; 281: 39-49.

3. Tedesco FS, Dellavalle A, Diaz-Manera J, Messina G, Cossu G. Repairing skeletal muscle: regenerative potential of skeletal muscle stem cells. J Clin Invest 2010; 120: $11-19$.

4. Jackson WM, Aragon AB, Bulken-Hoover JD, Nesti LJ, Tuan RS. Putative heterotopic ossification progenitor cells derived from traumatized muscle. J Orthop Res 2009; 27: 1645-1651.

5. Zundel WS, Tyler FH. The muscular dystrophies. N Engl J Med 1965; 273: 596-601; CONCL.

6. Frank NY, Kho AT, Schatton T, Murphy GF, Molloy MJ, Zhan $Q$ et al. Regulation of myogenic progenitor proliferation in human fetal skeletal muscle by BMP4 and its antagonist Gremlin. J Cell Biol 2006; 175: 99-110.

7. Ono Y, Calhabeu F, Morgan JE, Katagiri T, Amthor $\mathrm{H}$, Zammit PS. BMP signalling permits population expansion by preventing premature myogenic differentiation in muscle satellite cells. Cell Death Differ 2011; 18: 222-234.

8. Clever JL, Sakai Y, Wang RA, Schneider DB. Inefficient skeletal muscle repair in inhibitor of differentiation knockout mice suggests a crucial role for BMP signaling during adult muscle regeneration. Am J Physiol Cell Physiol 2010; 298: C1087-C1099.

9. Bandyopadhyay A, Tsuji K, Cox K, Harfe BD, Rosen V, Tabin CJ. Genetic analysis of the roles of BMP2, BMP4, and BMP7 in limb patterning and skeletogenesis. PLOS Genet 2006; 2: e216.

10. Dahlqvist C, Blokzijl A, Chapman G, Falk A, Dannaeus K, Ibanez CF et al. Functional Notch signaling is required for BMP4-induced inhibition of myogenic differentiation. Development 2003; 130: 6089-6099.

11. Polesskaya A, Seale $P$, Rudnicki MA. Wnt signaling induces the myogenic specification of resident CD45 + adult stem cells during muscle regeneration. Cell 2003; 113: 841-852.

12. Bernardi H, Gay S, Fedon Y, Vernus B, Bonnieu A, Bacou F. Wnt4 activates the canonical beta-catenin pathway and regulates negatively myostatin: functional implication in myogenesis. Am J Physiol Cell Physiol 2011; 300: C1122-C1138.

13. Sethi JK, Vidal-Puig A. Wnt signalling and the control of cellular metabolism. Biochem $J$ 2010; 427: 1-17.

14. Guo X, Wang XF. Signaling cross-talk between TGF-beta/BMP and other pathways. Cell Res 2009; 19: 71-88.

15. Hu MC, Rosenblum ND. Smad1, beta-catenin and Tcf4 associate in a molecular complex with the Myc promoter in dysplastic renal tissue and cooperate to control Myc transcription. Development 2005; 132: 215-225.

16. Labbe E, Letamendia A, Attisano L. Association of Smads with lymphoid enhancer binding factor $1 / T$ cell-specific factor mediates cooperative signaling by the transforming growth factor-beta and wnt pathways. Proc Natl Acad Sci USA 2000; 97: 8358-8363. 
17. Hannenhalli S, Kaestner $\mathrm{KH}$. The evolution of Fox genes and their role in development and disease. Nat Rev Genet 2009; 10: 233-240.

18. Nifuji A, Miura N, Kato N, Kellermann O, Noda M. Bone morphogenetic protein regulation of forkhead/winged helix transcription factor Foxc2 (Mfh1) in a murine mesodermal cell line C1 and in skeletal precursor cells. J Bone Miner Res 2001; 16: 1765-1771.

19. Davis KE, Moldes M, Farmer SR. The forkhead transcription factor FoxC2 inhibits white adipocyte differentiation. J Biol Chem 2004; 279: 42453-42461.

20. Perez VA, Ali Z, Alastalo TP, Ikeno F, Sawada H, Lai YJ et al. BMP promotes motility and represses growth of smooth muscle cells by activation of tandem Wnt pathways. J Cell Biol 2011; 192: 171-188.

21. Habas R, Kato $\mathrm{Y}, \mathrm{He} \mathrm{X}$. Wnt/Frizzled activation of Rho regulates vertebrate gastrulation and requires a novel Formin homology protein Daam1. Cell 2001; 107: 843-854.

22. Nishiyama T, Kii I, Kudo A. Inactivation of Rho/ROCK signaling is crucial for the nuclear accumulation of FKHR and myoblast fusion. J Biol Chem 2004; 279: 47311-47319.

23. Fortier M, Comunale F, Kucharczak J, Blangy A, Charrasse S, Gauthier-Rouviere C. RhoE controls myoblast alignment prior fusion through RhoA and ROCK. Cell Death Differ 2008; 15: 1221-1231.

24. Retting KN, Song B, Yoon BS, Lyons KM. BMP canonical Smad signaling through Smad1 and Smad5 is required for endochondral bone formation. Development 2009; 136 1093-1104.

25. Bryson-Richardson R, JCurrie PD. The genetics of vertebrate myogenesis. Nat Rev Genet 2008; 9: 632-646.

26. Daczewska M, Picchio L, Jagla T, Figeac N, Jagla K. Muscle development and regeneration in normal and pathological conditions: learning from Drosophila. Curr Pharm Des 2010; 16: 929-941.

27. Amthor $\mathrm{H}$, Christ $\mathrm{B}$, Weil M, Patel K. The importance of timing differentiation during limb muscle development. Curr Biol 1998; 8: 642-652.

28. Zhao P, Hoffman EP. Embryonic myogenesis pathways in muscle regeneration. Dev Dyn 2004; 229: 380-392.

29. Brack AS, Conboy IM, Conboy MJ, Shen J, Rando TA. A temporal switch from notch to Wnt signaling in muscle stem cells is necessary for normal adult myogenesis. Cell Stem Cell 2008; 2: 50-59.

30. Chang J, Sonoyama W, Wang Z, Jin Q, Zhang C, Krebsbach PH et al. Noncanonical Wnt-4 signaling enhances bone regeneration of mesenchymal stem cells in craniofacial defects through activation of p38 MAPK. J Biol Chem 2007; 282: 30938-30948.

31. Trompouki E, Bowman TV, Lawton LN, Fan ZP, Wu DC, DiBiase A et al. Lineage regulators direct BMP and Wnt pathways to cell-specific programs during differentiation and regeneration. Cell 2011; 147: 577-589.
32. Fukuda T, Kokabu S, Ohte S, Sasanuma H, Kanomata K, Yoneyama K et al. Canonical Wnts and BMPs cooperatively induce osteoblastic differentiation through a GSK3beta-dependent and beta-catenin-independent mechanism. Differentiation 2010; 80 : 46-52.

33. McBeath R, Pirone DM, Nelson CM, Bhadriraju K, Chen CS. Cell shape, cytoskeletal tension, and RhoA regulate stem cell lineage commitment. Dev Cell 2004; 6: 483-495.

34. Li G, Peng H, Corsi K, Usas A, Olshanski A, Huard J. Differential effect of BMP4 on NIH/ 3T3 and $\mathrm{C} 2 \mathrm{C} 12$ cells: implications for endochondral bone formation. J Bone Miner Res 2005; 20: 1611-1623.

35. Friedrichs M, Wirsdoerfer F, Flohe SB, Schneider S, Wuelling M, Vortkamp A. BMP signaling balances proliferation and differentiation of muscle satellite cell descendants. BMC Cell Biol 2011; 12: 26.

36. Hannallah D, Peng H, Young B, Usas A, Gearhart B, Huard J. Retroviral delivery of Noggin inhibits the formation of heterotopic ossification induced by BMP-4, demineralized bone matrix, and trauma in an animal model. J Bone Joint Surg Am 2004; 86-A: 80-91.

37. Liu K, Tripp S, Layfield LJ. Heterotopic ossification: review of histologic findings and tissue distribution in a 10-year experience. Pathol Res Pract 2007; 203: 633-640.

38. Weber FE, Schmokel H, Oelgeschlager M, Nickel J, Maly FE, Hortschansky P et al. Deletion mutants of BMP folding variants act as BMP antagonists and are efficient inhibitors for heterotopic ossification. J Bone Miner Res 2003; 18: 2142-2151.

39. Gannon FH, Kaplan FS, Olmsted E, Finkel GC, Zasloff MA, Shore E. Bone morphogenetic protein $2 / 4$ in early fibromatous lesions of fibrodysplasia ossificans progressiva. Hum Pathol 1997; 28: 339-343.

40. Kan L, Kessler JA. Animal models of typical heterotopic ossification. J Biomed Biotechnol 2011; 2011: 309287.

41. Hong JH, Hwang ES, McManus MT, Amsterdam A, Tian Y, Kalmukova R et al. TAZ, a transcriptional modulator of mesenchymal stem cell differentiation. Science 2005; 309 : 1074-1078.

42. Veeman MT, Slusarski DC, Kaykas A, Louie SH, Moon RT. Zebrafish prickle, a modulator of noncanonical Wnt/Fz signaling, regulates gastrulation movements. Curr Biol 2003; 13: $680-685$.

43. Miao J, Mu D, Ergel B, Singavarapu R, Duan Z, Powers S et al. Hepsin colocalizes with desmosomes and induces progression of ovarian cancer in a mouse model. Int $\mathrm{J}$ Cancer 2008; 123: 2041-2047.

44. Huang da W, Sherman BT, Lempicki RA. Systematic and integrative analysis of large gene lists using DAVID bioinformatics resources. Nat Protoc 2009; 4: 44-57. 